\title{
Higher order modular forms and mixed Hodge theory
}

\author{
by \\ RAmesh SReekantan (Bangalore)
}

Introduction. In the theory of automorphic forms, the classical holomorphic modular forms and their complex conjugates play a special role: they are the forms most closely linked to geometry. Spaces of such modular forms can be identified with the cohomology groups of certain locally constant sheaves on modular curves. From this point of view certain things, like Hecke operators, become very natural and this is the first step in associating a motive to a modular form.

Second order modular forms were introduced by Goldfeld and have been studied in some detail by several people. Examples of such forms we also discovered in a different context by Kleban and Zagier [KZ03]. In [CDO02], the notion of a higher order automorphic form was considered and the spaces of such forms were studied. One can speculate as to whether there is any geometry underlying these spaces of automorphic forms. In general, these spaces can be rather large and one cannot expect much. However, in this paper we consider certain subspaces with additional structure that can be understood as the generalization of the space of classical holomorphic modular forms. We show that these spaces can be identified with certain spaces coming from the fundamental groups of modular curves.

In weight 0 , the spaces we consider are the spaces of anti-derivatives of iterated integrals of smooth 1 -forms. In the classical situation the EichlerShimura isomorphism identifies the spaces of holomorphic modular forms along with the conjugates of cusp forms with the cohomology groups of locally constant sheaves on a modular curve and these spaces have mixed Hodge structures. We show that our spaces can be identified with graded quotients of the dual of the group ring of the fundamental group of the modular curve generalizing the Eichler-Shimura theorem. These quotients have mixed Hodge structures due to Hain [Hai87] and Morgan. Using this,

2010 Mathematics Subject Classification: 11F11, 14C30, 30F30, 32S35.

Key words and phrases: higher order modular forms, Chen iterated integrals, mixed Hodge structures, Poincaré series. 
we can define a mixed Hodge structure on the space of weight 0 higher order forms. In general, this Hodge structure depends on the base point. Deligne and Goncharov [DG05] show that this Hodge structure is the same as that on a cohomology group of a pair.

For higher weights, we generalize a construction of Goldfeld and O'Sullivan [GO03] using twisted Poincaré series to construct some higher order higher weight forms. We show that, when the weight $k$ is greater than 2 , the spaces of such forms also have a mixed Hodge structure.

One purpose of relating the higher order forms to the geometry of the modular curve is to define a Hecke theory. Classically, the Hecke operators are the operators induced on the cohomology groups by certain algebraic correspondences. In the higher order case, however, one cannot use this, as Hecke correspondences do not induce maps between homotopy groups or on the corresponding Hodge structures. This perhaps explains why there is no satisfactory Hecke theory for higher order forms.

In weight 0 , much of the theory is analogous to the theory of multiple zeta values and the geometry of $\mathbb{P}^{1}-\{0,1, \infty\}$ due to Deligne, Goncharov [DG05] and others. From this point of view the higher order modular forms can be viewed as generalizations of the single variable multiple polylogarithms.

1. Higher order automorphic forms. Let $\Gamma$ be a discrete subgroup of $\mathrm{SL}_{2}(\mathbb{R})$ with no elliptic fixed points, so $\Gamma$ is isomorphic to the fundamental group $\pi_{1}\left(X, x_{0}\right)$ of $X=\Gamma \backslash \mathfrak{H}$ at some point $x_{0} \in X$. Let $\mathbb{Z}[\Gamma]$ be the group ring and $J=J_{x_{0}}=\langle\gamma-1\rangle$ the augmentation ideal of $\mathbb{Z}[\Gamma]$ which fits in the exact sequence

$$
0 \rightarrow J \rightarrow \mathbb{Z}[\Gamma] \stackrel{\text { deg }}{\longrightarrow} \mathbb{Z} \rightarrow 0 .
$$

If $f: \mathfrak{H} \rightarrow \mathbb{C}$ is a function and $k \in \mathbb{Z}$, define

$$
\left(\left.f\right|_{k} \gamma\right)(z)=j(\gamma, z)^{-k} f(\gamma z)
$$

where $\gamma=\left(\begin{array}{ll}a & b \\ c & d\end{array}\right)$ is in $\mathrm{SL}_{2}(\mathbb{R})$ and $j(\gamma, z)=(c z+d)$ is the usual automorphy factor. We extend this to an action of $\mathbb{Z}[\Gamma]$ on the space of functions by defining

$$
\left.f\right|_{k} \sum a_{i} \gamma_{i}=\left.\sum a_{i} f\right|_{k} \gamma_{i}
$$

An automorphic form of weight $k$ for $\Gamma$ is a function such that

$$
\left.f\right|_{k} \gamma=0
$$

for all $\gamma$ in $J$. More generally, for $s \in \mathbb{N}$, we define a higher order automorphic form of weight $k$ and order $s$ for $\Gamma$ to be a function $f: \mathfrak{H} \rightarrow \mathbb{C}$ such that

$$
\left.f\right|_{k} \gamma=0
$$

for all $\gamma$ in $J^{s}$. Let $M_{k}^{s}=M_{k}^{s}(\Gamma)$ denote the space of higher order automorphic forms of order $s$. We further define $M_{k}^{0}(\Gamma)$ to be the constants $\mathbb{C}$. For 
$s=1$ with the added conditions of holomorphy and growth at the cusps, this gives the classical modular forms of weight $k$. For a fixed weight $k$ there is a natural filtration by order

$$
M_{k}^{0} \subseteq M_{k}^{1} \subseteq M_{k}^{2} \subseteq \cdots .
$$

These spaces can be rather large, so one cannot expect much structure. In this paper we study certain subspaces of the space of these forms which have a lot more structure.

2. Iterated integrals. Let $X$ be a smooth manifold with a point $x_{0}$. Let $P(X)=P\left(X, x_{0}\right)$ denote the space of loops on $X$ based at $x_{0}$, that is, continuous functions

$$
\gamma:[0,1] \rightarrow X, \quad \gamma(0)=\gamma(1)=x_{0} .
$$

A function $\phi: P(X) \rightarrow R$, where $R$ is a ring, is said to be a homotopy functional if $\phi$ depends only on the homotopy class of $\gamma$. That is, it defines a function on $\Gamma=\pi_{1}\left(X, x_{0}\right)$ or equivalently an element of $\operatorname{Hom}_{\mathrm{AbGrps}}(\mathbb{Z}[\Gamma], R)$.

Let $E^{\bullet}(X)$ denote the de Rham complex of smooth forms on $X$. It is a differential graded algebra (dga), that is, a graded algebra $A$ with a degree 1 map $d: A \rightarrow A$ such that

- $d \circ d=0$,

- $d(a \cdot b)=d(a) \cdot b+(-1)^{\operatorname{deg}(a)} a \cdot d(b)$.

Let $\omega$ be a 1 -form in $\mathcal{A}^{1}(X)$, where $\mathcal{A}^{\bullet}(X)$ is a sub-dga of $E^{\bullet}(X)$. The map

$$
\gamma \mapsto \int_{\gamma} \omega=\int_{0}^{1} f(t) d t,
$$

where $\gamma^{*}(\omega)=f(t) d t$, defines a function on $P(X)$. This defines an element of $\operatorname{Hom}(\mathbb{Z}[\Gamma], \mathbb{R})$ if and only if $\omega$ is closed. Hence this only detects elements of $\Gamma$ visible in the homology of $X$. It vanishes on $J^{2}$, since if $(\alpha-1)(\beta-1) \in J^{2}$, then

$$
\int_{\alpha \beta} \omega=\int_{\alpha} \omega+\int_{\beta} \omega
$$

so

$$
\int_{\alpha \beta} \omega-\int_{\alpha} \omega-\int_{\beta} \omega+\int_{1} \omega=0 .
$$

The iterated integrals studied by Chen [Che71] detect more elements of the group ring. Suppose $\omega_{1}, \ldots, \omega_{r}$ are smooth 1-forms in $\mathcal{A}^{1}(X)$ and $\gamma$ is a path on $X$. Define

$$
\int_{\gamma} \omega_{1} \ldots \omega_{r}=\int_{0 \leq t_{1} \leq \cdots \leq t_{r} \leq 1} \ldots f_{1}\left(t_{1}\right) \ldots f_{r}\left(t_{r}\right) d t_{1} \ldots d t_{r}
$$


where $\gamma^{*}\left(\omega_{i}\right)=f_{i}(t) d t$. This defines a function on the space of paths of $X$ which will be denoted by $\int \omega_{1} \ldots \omega_{r}$ and is called an iterated line integral of length $r$. A linear combination of such functions is called an iterated integral and its length is the length of the longest line integral. Length 0 iterated integrals are defined to be constant functions. Let $B_{s}\left(\mathcal{A}^{\bullet}(X)\right)$ denote the space of iterated integrals of length $\leq s$ coming from forms in $\mathcal{A}^{\bullet}$.

An iterated integral is not necessarily invariant under homotopy. Chen [Che71] formulated a condition in terms of differential graded algebras under which iterated integrals which are homotopy functionals are closed with respect to a certain differential. However, we have no use for that formalism in what follows so we will not describe it. It does underlie the following notation though. Let $H^{0}\left(B_{s}\left(\mathcal{A}^{\bullet}(X)\right), x_{0}\right)$ be the space of iterated integrals of length $\leq s$ which are homotopy functionals on loops based at a point $x_{0}$ on $X$ modulo those iterated integrals which integrate to 0 along any path. We shorten this to $H^{0}\left(B_{s}(X), x_{0}\right)$ if $\mathcal{A}^{\bullet}(X)$ is $E^{\bullet}(X)$.

If $I$ is in $B_{s}\left(\mathcal{A}^{\bullet}(X)\right)$ and $\gamma$ in $\Gamma$, let

$$
\langle I, \gamma\rangle=\int_{\gamma} I
$$

denote the evaluation map. This can be extended by linearity to all of $\mathbb{Z}[\Gamma]$. Let $H^{0}\left(\bar{B}_{s}\left(\mathcal{A}^{\bullet}(X)\right), x_{0}\right)$ denote the subspace of $H^{0}\left(B_{s}\left(\mathcal{A}^{\bullet}(X)\right), x_{0}\right)$ such that

$$
\left\langle I, \eta_{x_{0}}\right\rangle=0
$$

where $\eta_{x_{0}}$ denotes the constant loop at $x_{0}$. Namely, these are iterated integrals with constant term being 0 .

We have the following propositions that can be found in [Hai87]. As we will have to appeal to them several times we find it useful to repeat them here.

Proposition 2.1 (Hain [Hai87, Proposition 2.9]). Let $\int \omega_{1} \ldots \omega_{s}$ be an iterated line integral and $\alpha$ and $\beta$ two paths such that $\alpha(1)=\beta(0)$. Then

$$
\int_{\alpha \beta} \omega_{1} \ldots \omega_{s}=\sum_{i=0}^{s} \int_{\alpha} \omega_{1} \ldots \omega_{i} \int_{\beta} \omega_{i+1} \ldots \omega_{s}
$$

where an empty integral is to be understood as 1.

As a corollary one has

Corollary 2.2. If $\alpha_{i}$ are loops and $\beta(0)=\alpha_{i}(1)$, then

$$
\int_{\prod_{k}\left(\alpha_{k}-1\right) \beta} \omega_{1} \ldots \omega_{s}=\sum_{i=1}^{s} \int_{\prod_{k}\left(\alpha_{k}-1\right)} \omega_{1} \ldots \omega_{i} \int_{\beta} \omega_{i+1} \ldots \omega_{s} .
$$


Let

$$
p_{s}: H^{0}\left(B_{s}(X), x_{0}\right) \rightarrow \bigotimes^{s} H^{1}(X, \mathbb{C})
$$

be defined as follows. For $\alpha_{1} \otimes \cdots \otimes \alpha_{s} \in \otimes H_{1}(X, \mathbb{C})$, where $\alpha_{i}$ are loops based at $x_{0}$,

$$
p_{s}(I)\left(\bigotimes_{i=1}^{s} \alpha_{i}\right)=\left\langle I, \prod\left(\alpha_{i}-1\right)\right\rangle .
$$

Proposition 2.3 (Hain [Hai87, Props. 2.10, 2.13]). If $\omega_{1} \ldots \omega_{r}$ are smooth one-forms on $X$ and $\alpha_{1}, \ldots, \alpha_{s}$ are loops based at $x_{0}$ then

$$
\left\langle\int \omega_{1} \ldots \omega_{r}, \prod_{i=1}^{s}\left(\alpha_{i}-1\right)\right\rangle= \begin{cases}\prod_{i=1}^{s} \int_{\alpha_{j}} \omega_{j} & \text { if } r=s \\ 0 & \text { if } r<s .\end{cases}
$$

Finally, we state another proposition which allows us to reduce the length of an iterated integral if one of the terms is exact.

Proposition 2.4 (Hain [Hai87, Prop. 1.3]). Suppose $\omega_{1}, \ldots, \omega_{s}$ are 1forms on $X$ and $\gamma$ is a path on $X$. If $f$ is a function on $M$ then

$$
\begin{aligned}
\int_{\gamma} d f \omega_{1} \ldots \omega_{s} & =\int_{\gamma}\left(f \omega_{1}\right) \omega_{2} \ldots \omega_{s}-f(\gamma(0)) \int_{\gamma} \omega_{1} \ldots \omega_{s}, \\
\int_{\gamma} \omega_{1} \ldots \omega_{i-1} d f \omega_{i} \ldots \omega_{s} & =\int_{\gamma} \omega_{1} \ldots\left(f \omega_{i}\right) \omega_{i+1} \ldots \omega_{s}-\int_{\gamma} \omega_{1} \ldots\left(f \omega_{i-1}\right) \omega_{i} \ldots \omega_{s}, \\
\int_{\gamma} \omega_{1} \ldots \omega_{s} d f & =f(\gamma(1)) \int_{\gamma} \omega_{1} \ldots \omega_{s}-\int_{\gamma} \omega_{1} \ldots \omega_{s-1}\left(f \omega_{s}\right) .
\end{aligned}
$$

This shows there exist non-trivial iterated integrals which integrate to 0 along any path.

3. Higher order modular forms of geometric origin. Now let $X=$ $\Gamma \backslash \mathfrak{H}$ where $\Gamma$ is an arithmetic subgroup of $\mathrm{SL}_{2}(\mathbb{R})$, and let $\pi: \mathfrak{H} \rightarrow X$ be the canonical map. Let $x_{0}$ be a point on $X$. We further assume that $\Gamma$ has no elliptic fixed points, so $\Gamma \simeq \pi_{1}\left(X, x_{0}\right)$. In this section we define a subspace of the space of higher order automorphic forms of weight 0 for $\Gamma$ which has an additional structure coming from the geometry of the curve $X$. As $X$ is a curve, it has the cuspidal compactification $\bar{X}=\Gamma \backslash\left(\mathfrak{H} \cup \mathbb{P}^{1}(\mathbb{Q})\right)$, which is a smooth projective curve. We also define a subspace of this space coming from $\bar{X}$ which generalize the classical cusp forms. Let $D=\Gamma \backslash \mathbb{P}^{1}(\mathbb{Q})$ be the set of cusps; then $\bar{X}=X \cup D$.

We have the following theorem.

TheOREM 3.1. Let I be an iterated integral on $X$ of length $\leq s$ which is a homotopy functional. Let $x_{0}$ denote a point on $X$. Let $z_{0}$ denote a point 
of $\mathfrak{H}$ lying in the fibre over $x_{0}$. Then the function $F_{I}$ on $\mathfrak{H}$ defined by

$$
F_{I}(z)=\int_{z_{0}}^{z} \pi^{*}(I):=\int_{z_{0}}^{z} I
$$

is a higher order modular form of order $s+1$. Further, this gives a well defined injective linear map

$$
H^{0}\left(B_{s}(X), x_{0}\right) \stackrel{\Psi}{\rightarrow} M_{0}^{s+1}(\Gamma) .
$$

Here and from now on, by abuse of notation, we use $I$ to denote both the integral on $X$ as well as its pullback $\pi^{*}(I)$ on $\mathfrak{H}$. As before we define an empty integral to be 1 and hence integrals of iterated integrals of length 0 , i.e. constants, are just constants.

Proof ot Theorem 3.1. Let $I$ be homotopy functional of length $\leq s$. This has an expression of the form

$$
I=\sum_{|J| \leq s} \omega_{J}
$$

where $\int \omega_{J}=\int \omega_{j_{1}} \ldots \omega_{j_{r}}$ are iterated line integrals. We need to show that for any $\gamma_{1}, \ldots, \gamma_{s+1} \in \Gamma$,

$$
\left.F_{I}\right|_{\prod_{i=1}^{s+1}\left(\gamma_{i}-1\right)}(z)=0 .
$$

Let $\eta$ be a path from $z_{0}$ to $z$ on $\mathfrak{H}$. If $\gamma$ is a loop on $X$ based at $x_{0}$, then one has a composite path $\gamma \pi(\eta)$ on $X$ from $x_{0}$ to $\pi(z)$. This can be lifted to a unique path on $\mathfrak{H}$ from $z_{0}$ to $\gamma z$ passing through $\gamma z_{0}$. We denote it by $\gamma \eta$.

Notice that

$$
\left.F_{I}\right|_{\gamma_{1}-1}(z)=\int_{z_{0}}^{\gamma_{1} z} I-\int_{z_{0}}^{z} I=\int_{\gamma_{1} \eta} I-\int_{\eta} I=\int_{\left(\gamma_{1}-1\right) \eta} I
$$

as

$$
\int_{\gamma z_{0}}^{\gamma z} I=\int_{z_{0}}^{z} I
$$

since $I$ is $\Gamma$-invariant.

For each iterated line integral $\int \omega_{J}=\int \omega_{j_{1}} \ldots \omega_{j_{r}}$ appearing in $I$ we can apply Corollary 2.2 and Proposition 2.3 to get

$$
\begin{aligned}
\int_{\prod_{i=1}^{s}\left(\gamma_{i}-1\right) \eta} \omega_{J} & =\sum_{i=1}^{s} \int_{\prod_{k=1}^{s}\left(\gamma_{i}-1\right)} \omega_{j_{1}} \ldots \omega_{j_{i}} \int_{\eta} \omega_{j_{i+1}} \ldots \omega_{j_{s}} \\
& = \begin{cases}\prod_{i=1}^{s} \int_{\gamma_{i}} \omega_{j_{i}} & \text { if } r=s \\
0 & \text { if } r<s .\end{cases}
\end{aligned}
$$


Therefore

$$
\left.F_{I}\right|_{\prod_{i=1}^{s}\left(\gamma_{i}-1\right)}(z)=\sum_{|J|=s} \prod_{i=1}^{s} \int_{\gamma_{i}} \omega_{j_{i}}
$$

and this expression is independent of $z$. In particular, applying $\gamma_{s+1}-1$ annihilates it, so $\left.F_{I}\right|_{J^{s+1}} \equiv 0$.

It should be remarked that since $I$ is a homotopy functional, $F_{I}(z)$ does not depend on the path from $z_{0}$ to $z$ but the iterated line integrals $\omega_{J}$ need not be homotopy invariant and hence we had to choose paths. However, by construction, the sums of iterated line integrals that appear in the expression of $\left.F_{I}\right|_{\gamma_{1}-1}(z)$ as a sum of products of iterated line integrals are homotopy functionals. That is, we can collect terms together in such a way that

$$
\int_{z_{0}}^{\gamma_{1} z} I-\int_{z_{0}}^{z} I=\int_{\left(\gamma_{1}-1\right) \eta} I=\sum_{r=1}^{s} \int_{z_{0}}^{\gamma_{1} z_{0}} I_{1}^{r} \int_{z_{0}}^{z} I_{2}^{r}
$$

where $I_{1}^{r}$ and $I_{2}^{r}$ are homotopy functionals. Note that $I_{2}^{s}=I_{1}^{0}=I$.

To prove injectivity, suppose $I$ is of order $s$ and $F_{I} \equiv 0$. Then in particular, for any $\gamma$ in $\Gamma$,

$$
F_{I}\left(\gamma z_{0}\right)=\int_{z_{0}}^{\gamma z_{0}} I=0
$$

so $I$ is 0 as a homotopy functional. Hence $I=0$ in $H^{0}\left(B_{s}(X), x_{0}\right)$.

3.1. The space of geometric higher order modular forms. A higher order automorphic form is said to be of geometric origin if it lies in the image of the map

$$
\Psi: H^{0}\left(B_{s}(X), x_{0}\right) \rightarrow M_{0}^{s+1}(\Gamma) .
$$

The space of such geometric higher order modular forms will be denoted by $M_{\text {geom, } 0}^{s+1}\left(\Gamma, x_{0}\right)$.

We have an inclusion map

$$
i: H^{0}\left(B_{s}(\bar{X}), x_{0}\right) \rightarrow H^{0}\left(B_{s}(X), x_{0}\right) .
$$

A geometric higher order modular form is said to be cuspidal if it lies in the image of

$$
\Psi \circ i: H^{0}\left(B_{s}(\bar{X}), x_{0}\right) \rightarrow M_{0}^{s+1}(\Gamma) .
$$

We denote the space of geometric cuspidal forms by $S_{\text {geom }, 0}^{s+1}\left(\Gamma, x_{0}\right)$.

If $K$ is in $H^{0}\left(B_{s}(\bar{X}), x_{0}\right)$ then for all $\sigma$ in $\Gamma_{\mathbf{a}}$, where $\Gamma_{\mathbf{a}}$ denotes the stabilizer of the cusp a,

$$
\int_{x_{0}}^{\sigma x_{0}} K=a
$$


where $a$ is a constant. This is because $K=I+a$, where $I$ is in $H^{0}\left(\bar{B}(\bar{X}), x_{0}\right)$ and $a$ is a constant, and

$$
\int_{x_{0}}^{\sigma x_{0}} I=0
$$

as the loop $\left\{x_{0}, \sigma x_{0}\right\}$ on $X$ is homotopic to the constant path on $\bar{X}$.

A consequence of this is the following lemma, which we will have occasion to use later.

Lemma 3.2. For $K$ in $H^{0}\left(B_{s}(\bar{X}), x_{0}\right)$ we have

$$
\int_{z_{0}}^{\sigma z} K-\int_{z_{0}}^{z} K=0
$$

for all parabolic $\sigma \in \Gamma_{\mathbf{a}}$ and all cusps $\mathbf{a}$. In particular, for a geometric higher order cusp form $f$ we have

$$
\left.f\right|_{\sigma-1} \equiv 0
$$

for all parabolic $\sigma \in \Gamma_{\mathbf{a}}$ and all cusps $\mathbf{a}$.

Proof. Any $K$ in $H^{0}\left(B_{s}(\bar{X}), x_{0}\right)$ is of the form $I+a$, where $a$ is a constant and $I$ is in $H^{0}\left(\bar{B}(\bar{X}), x_{0}\right)$. Hence

$$
\int_{z_{0}}^{\sigma z} K-\int_{z_{0}}^{z} K=\left(a+\int_{z_{0}}^{\sigma z} I\right)-\left(a+\int_{z_{0}}^{z} I\right)=\int_{z_{0}}^{\sigma z} I-\int_{z_{0}}^{z} I .
$$

From Proposition 2.1 we have

$$
\int_{z_{0}}^{\sigma z} I=\int_{z_{0}}^{z} I+\int_{z}^{\sigma z} I+\sum_{r=1}^{s-1} \int_{z_{0}}^{z} I_{r}^{\prime} \int_{z}^{\sigma z} I_{r}^{\prime \prime}
$$

where $I_{r}^{\prime}$ and $I_{r}^{\prime \prime}$ are certain iterated integrals which are homotopy functionals but of order between 1 and $s-1$. This calculation concerns forms on $\bar{X}$, so $I, I_{r}^{\prime}$ and $I_{r}^{\prime \prime}$ are all in $H^{0}\left(\bar{B}_{s}(\bar{X}), \pi(z)\right)$. Explicitly, this expansion comes from a repeated application of Proposition 2.1- so the forms which constitute the $I_{r}^{\prime} \mathrm{s}$, and $I_{r}^{\prime \prime} \mathrm{s}$ are the forms which constitute $I$, and $I$ is made up of forms on $\bar{X}$. Hence all the iterated integrals are in $\bar{B}_{s}(\bar{X})$. Since we also know they are homotopy invariant, they are in $H^{0}\left(\bar{B}_{s}(\bar{X}), \pi(z)\right)$.

Since $\sigma \in \Gamma_{\mathbf{a}}$ one has

$$
\int_{z}^{\sigma z} I=0 \text { and } \int_{z}^{\sigma z} I_{r}^{\prime \prime}=0,
$$

most of the terms vanish and

$$
\int_{z_{0}}^{\sigma z} I=\int_{z_{0}}^{z} I
$$


A geometric cusp form is of the form $f(z)=\int_{z_{0}}^{z} K$ for some $K$ in $H^{0}\left(B_{s}(\bar{X}), x_{0}\right)$ for some $s$. Hence for all $z$,

$$
\left.f\right|_{\sigma-1}(z)=\int_{z_{0}}^{\sigma z} K-\int_{z_{0}}^{z} K=0 .
$$

From the work of Chen [Che71] there is an isomorphism

$$
H^{0}\left(B_{s}\left(\mathcal{A}^{\bullet}(X)\right), x_{0}\right) \rightarrow \operatorname{Hom}\left(\mathbb{Q}\left[\pi_{1}\left(X, x_{0}\right)\right] / J^{s+1}, \mathbb{C}\right)
$$

and an isomorphism

$$
H^{0}\left(\bar{B}_{s}\left(\mathcal{A}^{\bullet}(X)\right), x_{0}\right) \rightarrow \operatorname{Hom}\left(J / J^{s+1}, \mathbb{C}\right)
$$

where $\mathcal{A}^{\bullet}$ is any complex quasi-isomorphic to the de Rham complex $E^{\bullet}$. Hence we can relate these spaces of modular forms to quotients of the group ring of the fundamental group of $X$. This motivates the phrase "geometric origin". Special cases of such forms were considered in [DS06b].

The second graded piece of $H^{0}\left(\bar{B}_{s}(X), x_{0}\right)$ is isomorphic to the first cohomology group of the modular curve $X$ :

$$
\operatorname{Hom}\left(J / J^{2}, \mathbb{C}\right) \simeq H^{1}(X, \mathbb{C}),
$$

and this corresponds to the fact that the space of classical modular forms of weight 2 is isomorphic to the space of second order modular forms of weight 0 via the map $f \mapsto F(z)=\int_{z_{0}}^{z} f(t) d t$. Similarly, the classical cusp forms of weight 2 correspond to cusp forms of weight 0 and exact order 2 via the same map - though in that case $J$ is the augmentation ideal of $\pi_{1}\left(\bar{X}, x_{0}\right)$.

More generally, one can consider the completion of the group ring with respect to the augmentation ideal

$$
\hat{\mathbb{Q}}\left[\pi\left(X, x_{0}\right)\right]=\lim _{s} \mathbb{Q}\left[\pi\left(X, x_{0}\right)\right] / J^{s} .
$$

This is called the Mal'tsev completion of the group ring. The space of all modular forms of weight 0 and geometric origin can be interpreted as the dual of this space.

3.2. Hodge structures. Hain (and independently, Morgan) showed that the quotients of the group ring with respect to powers of the augmentation ideal $J$ have a mixed Hodge structure.

Proposition 3.3 (Hain and Morgan, [Hai87]). If $X$ is an algebraic variety over $\mathbb{C}$ and $x_{0}$ is a point on $X$, then there is a mixed Hodge structure on the space

$$
H^{0}\left(B_{s}(X), x_{0}\right)=\operatorname{Hom}\left(\mathbb{Q}\left[\pi_{1}\left(X, x_{0}\right)\right] / J^{s+1}, \mathbb{C}\right)
$$

which is natural with respect to morphisms of pointed varieties. Further, if $X$ is smooth and projective, then the length and weight filtrations coincide. 
In particular, this holds for the algebraic curves $X=\Gamma \backslash \mathfrak{H}$ and their compactifications $\bar{X}$. Hence the spaces of geometric higher order modular forms $M_{\text {geom, } 0}^{s+1}\left(\Gamma, x_{0}\right)$ and cusp forms $S_{\text {geom }, 0}^{s+1}\left(\Gamma, x_{0}\right)$ also inherit mixed Hodge structures.

A rough outline of how the Hodge structure is obtained is as follows, at least when $X$ is a smooth curve as in our case. Essentially the same procedure works for a smooth quasi-projective variety. We follow Hain [Hai87]. Let $\bar{X}$ denote the smooth compactification as above and $D=\bar{X}-X$. Let $E^{\bullet}(X \log D)$ denote the $\log$-complex of smooth forms with $\log$ singularities. This complex is quasi-isomorphic to the de Rham complex $E_{\mathbb{C}}^{\bullet}(X)$, hence the result of Chen [Che71] implies that all homotopy functionals can be obtained by iterated integrals of such forms. So it suffices to use such forms to define the Hodge structure.

One first defines the Hodge structure on the log-complex as follows:

$$
\begin{aligned}
& F^{p} E^{\bullet}(X \log D)=\left\{\text { forms with } \geq p \text { differentials } d z^{\prime}\right\}, \\
& W_{l} E^{\bullet}(X \log D)=\left\{\text { forms with } \leq l \text { quotients } \frac{d z^{\prime}}{z}\right\},
\end{aligned}
$$

and Deligne [Del71] showed that this induces a Hodge structure on the cohomology of $X$ by defining the Hodge and weight filtrations to be the image of the cohomology of these filtrations in the cohomology of $X$.

Define the filtrations on $B_{s}\left(E^{\bullet}(X \log D)\right)$ as follows:

$$
\begin{gathered}
F^{p} B_{s}\left(E^{\bullet}(X \log D)\right)=\operatorname{span}\left\{\int \omega_{1} \ldots \omega_{r} \mid \omega_{i} \in F^{p_{i}} \text { and } \sum_{i=1}^{r} p_{i} \geq p\right\}, \\
W_{l} B_{s}\left(E^{\bullet}(X \log D)\right)=\operatorname{span}\left\{\int \omega_{1} \ldots \omega_{r} \mid \omega_{i} \in W_{l_{i}} \text { and } r+\sum_{i=1}^{r} l_{i} \leq l\right\} .
\end{gathered}
$$

The Hodge and weight filtrations on $B_{s}\left(E^{\bullet}(X \log D)\right)$ induce filtrations on $H^{0}\left(B_{s}\left(E^{\bullet}(X \log D)\right)\right)$ and these define a mixed Hodge structure. Using the map $\Psi$ we get a mixed Hodge structure on the space of geometric higher order modular forms of weight 0 .

If $X=\bar{X}$ is a smooth projective curve then the weight filtration on $E^{1}(\bar{X})$ is given by

$$
0=W_{-1} \subset W_{0}=E^{1}(\bar{X}),
$$

hence

$$
W_{l} B_{s}\left(E^{\bullet}(\bar{X})\right)= \begin{cases}B_{l}\left(E^{\bullet}(\bar{X})\right) & \text { if } l \leq s \\ B_{s}\left(E^{\bullet}(\bar{X})\right) & \text { if } l \geq s\end{cases}
$$

Therefore

$$
G r_{s}^{W \bullet} H^{0}\left(B_{s}\left(E^{\bullet}(\bar{X})\right), x_{0}\right)=H^{0}\left(B_{s}\left(E^{\bullet}(\bar{X})\right), x_{0}\right) / H^{0}\left(B_{s-1}\left(E^{\bullet}(\bar{X})\right), x_{0}\right),
$$


so the filtration by length coincides with the weight filtration and the length graded pieces have a pure Hodge structure. Hence the space of geometric cusp forms of exact order $s$ has a pure Hodge structure.

In general that is not true, as for example, if $X$ is not compact and has more than one cusp,

$$
H^{0}\left(B_{1}(X), x_{0}\right) / H^{0}\left(B_{0}(X), x_{0}\right) \simeq H^{1}(X, \mathbb{C})
$$

does not have a pure Hodge structure, since the integral of an Eisenstein series lies in the weight 2 graded part of the weight filtration. So one sees that the space of higher order modular forms of weight 0 and order exactly 2 does not have a pure Hodge structure.

The Hodge structure generalizes the classical Eichler-Shimura Hodge structure on the space of classical modular forms of weight 2 as that can be identified with $H^{0}\left(\bar{B}_{1}(X), x_{0}\right)$. In this case the Hodge structure does not depend on the choice of $x_{0}$, but in general it does.

REMARK 3.4. More generally, one can construct the motive underlying this Hodge structure as the motive underlying the Hodge structure on the fundamental group is understood. This is described in the paper of Deligne and Goncharov [DG05, Proposition 3.4]. There they show that the Hodge structure on the graded pieces of the group ring of the fundamental group can be realized as the Hodge structure on the relative cohomology groups of pairs $\left(X^{s}, \bigcup_{i=0}^{s} X_{i}\right)$, where

- $X^{s}=X \times \cdots \times X$ ( $s$ times $)$;

- $X_{0}$ is the subvariety given by $t_{1}=x_{0}$, i.e. $x_{0} \times X^{s-1}$;

- $X_{i}$ is the subvariety given by $t_{i}=t_{i+1}$ for $0<i<s$, i.e. $X^{i-1} \times \Delta \times$ $X^{s-(i+1)}$, where $\Delta$ is the diagonal in $X \times X$ in the $i$ th and $(i+1)$ st places;

- $X_{s}$ is given by $t_{s}=x_{0}$, i.e. $X^{s-1} \times x_{0}$.

We have

$$
H^{s}\left(X^{s} / \bigcup_{i=0}^{s} X_{i}, \mathbb{C}\right) \simeq \operatorname{Hom}\left(J / J^{s+1}, \mathbb{C}\right)=H^{0}\left(\bar{B}_{s}(X), x_{0}\right)
$$

so precisely we get the space of weight 0 geometric modular forms of order $s$ modulo those of order 0 . For example, when $s=1$ we have

$$
H^{1}\left(X /\left\{x_{0}\right\}, \mathbb{C}\right) \simeq H^{1}(X, \mathbb{C}) \simeq \operatorname{Hom}\left(J / J^{2}, \mathbb{C}\right) .
$$

Hence the motive underlying the Hodge structure on the space of geometric higher order modular forms of weight 0 and order $s$ and base point $x_{0}$ is the motive associated to the pair $\left(X^{s}, \bigcup_{i=0}^{s} X_{i}\right)$. Namely, to this object one can associate de Rham, étale and Betti realization which are isomorphic when the field of coefficients is large enough. 
REMARK 3.5. Classically, one way of understanding Hecke operators is as follows. The space of classical modular forms of weight $k$ along with the complex conjugates of the cusp forms is, via the Eichler-Shimura map, identified with the cohomology of a local system on the modular curve, and this imposes a Hodge structure on this space. Hecke operators can then be understood as the morphisms of this Hodge structure induced by certain algebraic correspondences called Hecke correspondences.

One might hope that the same algebraic correspondences would induce morphisms on the Hodge structure of the space of geometric higher order modular forms of weight 0 , thus suggesting a way to define Hecke operators on these forms. However, unfortunately they do not, as, for example, they do not preserve base points and the Hodge structure does depend on the base point for $s>2$. Hence one cannot get notions of Hecke eigenfunctions or Hecke eigenspaces using Hecke correspondences and, as things stand, one cannot use this approach to define a motive of a higher order modular form.

3.2.1. Product structure. Let

$$
\hat{M}_{\text {geom }, 0}\left(X, x_{0}\right)=\underset{s}{\lim } M_{\text {geom }, 0}^{s}\left(X, x_{0}\right) .
$$

This space has a product structure induced by the product structure of iterated integrals

$$
B_{s_{1}}(X) \otimes B_{s_{2}}(X) \rightarrow B_{s_{1}+s_{2}-1}(X) .
$$

Explicitly, this is given by the shuffle product [Hai87, Lemma 2.11]. have

For example, for two closed 1-forms $\omega_{1}$ and $\omega_{2}$ with $F_{\omega_{i}}(z)=\int_{z_{0}}^{z} \omega_{i}$ we

$$
F_{\omega_{1}}(z) F_{\omega_{2}}(z)=F_{\omega_{1} \omega_{2}}(z)+F_{\omega_{2} \omega_{1}}(z) .
$$

4. Higher weights. We now consider the problem of constructing higher weight, higher order forms. Let $X$ and $x_{0}$ be as above. We have the following inductive definition for cuspidal higher order forms. A higher order form $f$ is said to be cuspidal if

- $\left.f\right|_{\gamma-1}$ is cuspidal;

- $\left.f\right|_{\phi-1} \equiv 0$ for all parabolic elements $\phi$ of $\Gamma$, that is,

$$
\phi \in \operatorname{Ker}\left\{\pi_{1}\left(X, x_{0}\right) \rightarrow \pi_{1}\left(\bar{X}, x_{0}\right)\right\} ;
$$

- $f$ satisfies a cuspidal growth condition: for all cusps a one has $\left.f\right|_{k}\left(\sigma_{\mathbf{a}}\right)(z) \ll e^{-c y}$ as $y \rightarrow \infty$ uniformly in $x$ for some constant $c>0$, where $\sigma_{\mathbf{a}}$ is an element of $\mathrm{SL}_{2}(\mathbb{Z})$ such that

$$
\sigma_{\mathbf{a}}(\infty)=\mathbf{a}
$$


A standard way of constructing classical modular forms is by Poincaré series. They are defined as follows. Let $\mathbf{a}$ be a cusp and $\sigma_{\mathbf{a}}$ as above, so the stabilizer of $\mathbf{a}$ is $\Gamma_{\mathbf{a}}=\sigma_{\mathbf{a}} \Gamma_{\infty} \sigma_{\mathbf{a}}^{-1}$. Let $m>0$ be an integer. For $k>2$, the Poincaré series $P_{m, \mathbf{a}}(z)=P_{m, \mathbf{a}, k}(z)$ of weight $k$ is defined as follows:

$$
P_{m, \mathbf{a}}(z)=\sum_{\gamma \in \Gamma_{\mathbf{a}} \backslash \Gamma} \frac{e\left(m \sigma_{\mathbf{a}}^{-1} \gamma z\right)}{j\left(\sigma_{\mathbf{a}}^{-1} \gamma, z\right)^{k}}
$$

where $e(z)=\exp (2 \pi i z)$. This is a cusp form. We can also define this when $m=0$, where this then gives the Eisenstein series of weight $k$ corresponding to the cusp a. The Poincaré series and Eisenstein series span the space of modular forms for $\Gamma$ as one varies $m$ and the cusps and in fact, for a fixed cusp, one can get a basis for the cusp forms by varying $m$ [Sar90].

We have the following generalization of Poincaré series, called twisted Poincaré series, which give rise to higher order forms. For order 2 this is due to Goldfeld and O'Sullivan ([Gol99], [GO03]). In what follows we suppress the weight $k$ in the notation.

Proposition 4.1. Let $k>2$ be an even integer and let $I$ be an element of $H^{0}\left(B_{s}(\bar{X}), x_{0}\right)$ and $z_{0}$ a point on $\mathfrak{H}$ lying in the fibre over $x_{0}$. Then, for every cusp a and non-negative integer $m$, we get three twisted Poincaré series:

$$
\begin{aligned}
& P_{m, \mathbf{a}}^{1}(z, I)=\sum_{\gamma \in \Gamma_{\mathbf{a}} \backslash \Gamma}\left(\int_{z_{0}}^{z} I\right) \frac{e\left(m \sigma_{\mathbf{a}}^{-1} \gamma z\right)}{j\left(\sigma_{\mathbf{a}}^{-1} \gamma, z\right)^{k}}, \\
& P_{m, \mathbf{a}}^{2}(z, I)=\sum_{\gamma \in \Gamma_{\mathbf{a}} \backslash \Gamma}\left(\int_{z_{0}}^{\gamma z_{0}} I\right) \frac{e\left(m \sigma_{\mathbf{a}}^{-1} \gamma z\right)}{j\left(\sigma_{\mathbf{a}}^{-1} \gamma, z\right)^{k}}, \\
& P_{m, \mathbf{a}}^{3}(z, I)=\sum_{\gamma \in \Gamma_{\mathbf{a}} \backslash \Gamma}\left(\int_{z}^{\gamma z} I\right) \frac{e\left(m \sigma_{\mathbf{a}}^{-1} \gamma z\right)}{j\left(\sigma_{\mathbf{a}}^{-1} \gamma, z\right)^{k}} .
\end{aligned}
$$

All of these are weight $k$, order $s+1$ modular forms and when $m>0$ they are cusp forms.

Further, there are relations between these modular forms coming from the fact that the integral of I over the path $\left\{z_{0}, \gamma z_{0}\right\}$ can be expressed, using Proposition 2.1, as a sum of products of integrals over $\left\{z_{0}, z\right\},\{z, \gamma z\}$ and $\left\{\gamma z, \gamma z_{0}\right\}$, where $\left\{z_{0}, z_{1}\right\}$ denotes a path from $z_{0}$ to $z_{1}$ in $\mathfrak{H}$ :

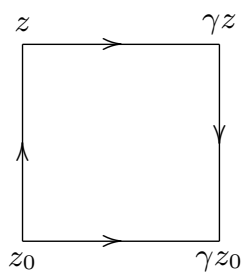


Proof. We first show that the summand is well defined. For $P^{1}$ there is no question. The arguments for $P^{2}$ and $P^{3}$ are similar and we have the following argument which we give for $P^{2}$.

It suffices to show that the term $\int_{z_{0}}^{\gamma z_{0}} I$ is well defined on $\Gamma_{\mathbf{a}} \backslash \Gamma$. Let $\sigma \in \Gamma_{\mathbf{a}}$ and $\gamma \in \Gamma$. Since $I$ is in $H^{0}\left(B_{s}(\bar{X}), x_{0}\right)$ and $\sigma \in \Gamma_{\mathbf{a}}$, from Lemma 3.2, we have

$$
\int_{z_{0}}^{\sigma z} I-\int_{z_{0}}^{z} I=0
$$

In particular, if $z=\gamma z_{0}$ we have

$$
\int_{z_{0}}^{\sigma \gamma z_{0}} I-\int_{z_{0}}^{\gamma z_{0}} I=0
$$

Hence the summand $\int_{z_{0}}^{\gamma z_{0}} I$ is well defined on $\Gamma_{\mathbf{a}} \backslash \Gamma$.

We now have a lemma regarding convergence.

Lemma 4.2. The series $P^{i}(z, I)$ converge for $k>2$.

Proof. For $i=1$, the series $P^{1}$ is simply a product of the classical Poincaré series and $\int_{z_{0}}^{z} I$, both of which converge for $k>2$.

For $P^{3}(z, I)$ the argument is similar to the case of $I=f$ in [DO06]. We can assume $I$ is in $H^{0}\left(\bar{B}_{s}(\bar{X}), x_{0}\right)$ since an element of $H^{0}\left(B_{s}(\bar{X}), x_{0}\right)$ differs from such an $I$ by a constant, and in the twisted Poincaré series this amounts to adding a constant multiple of a classical Poincaré series.

We use the estimate [DKMO06, Lemma 3]: for a classical cusp form $f$ of weight 2 , and any cusp a,

$$
\int_{z_{0}}^{z} f(w) d w \ll\left|\log \left(\operatorname{Im}\left(\sigma_{\mathbf{a}} z\right)\right)\right|+1 .
$$

To simplify exposition, we use the notation $y_{\mathbf{a}}(z)$ for $\operatorname{Im}\left(\sigma_{\mathbf{a}}(z)\right)$. Using the above estimate repeatedly, we deduce that for an iterated integral $I$ of length $r$,

$$
\int_{z_{0}}^{z} I \ll \frac{\left|\log ^{r}\left(y_{\mathbf{a}}(z)\right)\right|}{r !}+\frac{\left|\log ^{r-1}\left(y_{\mathbf{a}}(z)\right)\right|}{(r-1) !}+\cdots+1 .
$$

Observe that

$$
\begin{aligned}
\frac{\left|\log ^{r}\left(y_{\mathbf{a}}(z)\right)\right|}{r !}+\frac{\left|\log ^{r-1}\left(y_{\mathbf{a}}(z)\right)\right|}{(r-1) !}+\cdots+1 & <\exp \left(\left|\log \left(y_{\mathbf{a}}(z)\right)\right|\right) \\
& <y_{\mathbf{a}}(z)+y_{\mathbf{a}}(z)^{-1}
\end{aligned}
$$

as $\exp (|\log (x)|)=x$ or $x^{-1}$. Replacing $y_{\mathbf{a}}(z)$ by $y_{\mathbf{a}}(z)^{\epsilon}$, we have, for $0<\epsilon<1$, 
using the fact that $\epsilon^{r}<\epsilon$,

$$
\int_{z_{0}}^{z} I \ll \epsilon^{-r}\left(y_{\mathbf{a}}(z)^{\epsilon}+y_{\mathbf{a}}(z)^{-\epsilon}\right) .
$$

To apply this to the convergence of the twisted Poincaré series we need an estimate for $\int_{z}^{\gamma z} I$. We have

$$
\int_{z}^{\gamma z} I=\sum_{j=0}^{r} \int_{z}^{z_{0}} I_{j} \int_{z_{0}}^{\gamma z} I_{j}^{\prime}
$$

where $I_{j}$ and $I_{j}^{\prime}$ are iterated integrals of lengths $j$ and $r-j$ respectively. From (6) and (7) we have

$$
\left|\int_{z}^{\gamma z} I\right| \ll \epsilon^{-r}(r+1)\left(y_{\mathbf{a}}(z)^{\epsilon}+y_{\mathbf{a}}(z)^{-\epsilon}\right)\left(y_{\mathbf{a}}(\gamma z)^{\epsilon}+y_{\mathbf{a}}(\gamma z)^{-\epsilon}\right) .
$$

Therefore

$$
P_{\mathbf{a}}^{3}(z, I) \ll \sum_{\gamma \in \Gamma_{\mathbf{a}} \backslash \Gamma} \frac{\left(y_{\mathbf{a}}(z)^{\epsilon}+y_{\mathbf{a}}(z)^{-\epsilon}\right)\left(y_{\mathbf{a}}(\gamma z)^{\epsilon}+y_{\mathbf{a}}(\gamma z)^{-\epsilon}\right)}{j(\gamma, z)^{-k}} .
$$

We have $y_{\mathbf{a}}(\gamma z)=y_{\mathbf{a}}(z) / j(\gamma, z)^{2}$. Replacing $j(\gamma, z)^{-k}$ by $\left(y_{\mathbf{a}}(\gamma z) / y_{\mathbf{a}}(z)\right)^{k / 2}$ in the expression above, we get

$$
\begin{aligned}
P_{\mathbf{a}}^{3}(z, I) \ll & y_{\mathbf{a}}(z)^{\epsilon-k / 2}\left(\sum_{\gamma \in \Gamma_{\mathbf{a}} \backslash \Gamma} y_{\mathbf{a}}(\gamma z)^{k / 2+\epsilon}+\sum_{\gamma \in \Gamma_{\mathbf{a}} \backslash \Gamma} y_{\mathbf{a}}(\gamma z)^{k / 2-\epsilon}\right) \\
& +y_{\mathbf{a}}(z)^{-\epsilon-k / 2}\left(\sum_{\gamma \in \Gamma_{\mathbf{a}} \backslash \Gamma} y_{\mathbf{a}}(\gamma z)^{k / 2+\epsilon}+\sum_{\gamma \in \Gamma_{\mathbf{a}} \backslash \Gamma} y_{\mathbf{a}}(\gamma z)^{k / 2-\epsilon}\right) .
\end{aligned}
$$

The sum

$$
E_{\mathbf{a}}(z, s)=\sum_{\gamma \in \Gamma_{\mathbf{a}} \backslash \Gamma} y_{\mathbf{a}}(\gamma z)^{s}
$$

is the classical non-holomorphic Eisenstein series for the cusp a and is known to be absolutely convergent in the region $\operatorname{Re}(s)>1$. So as long as $k / 2-\epsilon>1$, that is, $k>2$, our series will converge.

Using the change of basepoint formula, $P^{2}(z, I)$ can be expressed as a finite linear combination of terms of the form $P^{1}\left(z, I^{\prime}\right) P^{3}\left(z, I^{\prime \prime}\right)$ for some iterated integrals $I^{\prime}$ and $I^{\prime \prime}$, so converges in the same region.

It remains to show the invariance property.

LEMMA 4.3. The $P^{i}(z, I)$ are higher order modular forms of weight $k$ and order $s+1$.

Proof. For $P^{1}$ this is immediate from the earlier proposition, as $\int_{z_{0}}^{z} I$ is a weight 0 order $s+1$ form, while $P(z)$ is order 1 , weight $k$, so the product is order $s+1$, weight $k$. For the second one, let $P(z, I)=P_{m, \mathbf{a}}^{2}(z, I)$. We 
will show that $\left.P(z, I)\right|_{\beta-1}$ can be expressed as a linear combination of terms of the form $P\left(z, I^{\prime}\right)$ where $I^{\prime}$ is an iterated integral which is a homotopy functional, but of length strictly less than that of $I$. The theorem will then follow by induction.

We have

$$
\begin{aligned}
P & (\beta z, I) j(\beta, z)^{-k}-P(z, I) \\
& =\sum_{\gamma \in \Gamma_{\mathbf{a}} \backslash \Gamma}\left(\int_{z_{0}}^{\gamma z_{0}} I\right) \frac{e\left(m \sigma_{\mathbf{a}}^{-1} \gamma \beta z\right)}{j\left(\sigma_{\mathbf{a}}^{-1} \gamma, \beta z\right)^{k} j(\beta, z)^{k}}-\sum_{\gamma \in \Gamma_{\mathbf{a}} \backslash \Gamma}\left(\int_{z_{0}}^{\gamma z_{0}} I\right) \frac{e\left(m \sigma_{\mathbf{a}}^{-1} \gamma z\right)}{j\left(\sigma_{\mathbf{a}}^{-1} \gamma, z\right)^{k}} \\
& =\sum_{\gamma \in \Gamma_{\mathbf{a}} \backslash \Gamma}\left(\int_{z_{0}}^{\gamma z_{0}} I\right) \frac{e\left(m \sigma_{\mathbf{a}}^{-1} \gamma \beta z\right)}{j\left(\sigma_{\mathbf{a}}^{-1} \gamma \beta, z\right)^{k}}-\sum_{\gamma \in \Gamma_{\mathbf{a}} \backslash \Gamma}\left(\int_{z_{0}}^{\gamma z_{0}} I\right) \frac{e\left(m \sigma_{\mathbf{a}}^{-1} \gamma z\right)}{j\left(\sigma_{\mathbf{a}}^{-1} \gamma, z\right)^{k}} \\
& =\sum_{\gamma \in \Gamma_{\mathbf{a}} \backslash \Gamma}\left(\int_{z_{0}}^{\gamma \beta^{-1} z_{0}} I\right) \frac{e\left(m \sigma_{\mathbf{a}}^{-1} \gamma z\right)}{j\left(\sigma_{\mathbf{a}}^{-1} \gamma, z\right)^{k}}-\sum_{\gamma \in \Gamma_{\mathbf{a}} \backslash \Gamma}\left(\int_{z_{0}}^{\gamma z_{0}} I\right) \frac{e\left(m \sigma_{\mathbf{a}}^{-1} \gamma z\right)}{j\left(\sigma_{\mathbf{a}}^{-1} \gamma, z\right)^{k}} .
\end{aligned}
$$

We can once more apply Proposition 2.1 with $\alpha=\left\{z_{0}, \gamma z_{0}\right\}$ and $\beta=$ $\left\{\gamma z_{0}, \gamma \beta^{-1} z_{0}\right\}$. Since $\left\{\gamma z_{0}, \gamma \beta^{-1} z_{0}\right\}$ is homotopic to $\left\{z_{0}, \beta^{-1} z_{0}\right\}$ on $\Gamma \backslash \mathfrak{H}$, and $I$ is homotopy invariant, we obtain

$$
\int_{z_{0}}^{\gamma \beta^{-1} z_{0}} I-\int_{z_{0}}^{\gamma z_{0}} I=\int_{z_{0}}^{\beta^{-1} z_{0}} I+\sum_{r=1}^{s-1} \int_{z_{0}}^{\gamma z_{0}} I_{1}^{r} \int_{z_{0}}^{\beta^{-1} z_{0}} I_{2}^{r},
$$

where $I_{1}^{r}$ and $I_{2}^{r}$ are sums of iterated line integrals appearing in the proof of Theorem 3.1. They are homotopy functionals by construction.

Combining this with (9) we have

$$
\begin{aligned}
P(\beta z, I) j(\beta, z)^{-k}-P & (z, I) \\
& =\left(\int_{z_{0}}^{\beta^{-1} z_{0}} I\right) P(z)+\sum_{r=1}^{s-1}\left(\int_{z_{0}}^{\beta^{-1} z_{0}} I_{2}^{r}\right) P\left(z, I_{1}^{r}\right) .
\end{aligned}
$$

This, by induction on $s$, is a higher order modular form of weight $k$ and order $s$. Hence $P(z, I)$ is a higher order modular form of weight $k$ and order $s+1$.

The third type of Poincaré series is a higher order modular form, as the iterated integral can be expressed in terms of the first two and products of lower order integrals using the change of basepoint formula which comes out of Proposition 2.1 and the fact that

$$
\int_{z_{0}}^{z} I=\int_{\gamma z_{0}}^{\gamma z} I
$$

for $\gamma \in \Gamma$. 
To show that $P_{m, \mathbf{a}}(z, I)$ is cuspidal for $m>0$, using (11) we find, by induction, that $\left.P_{m, \mathbf{a}}(z, I)\right|_{\beta-1}$ is cuspidal. Further, if $\phi$ is a parabolic element, then $\left.P_{m, \mathbf{a}}(z, I)\right|_{\phi-1}=0$ as $\int_{z_{0}}^{\phi z_{0}} I_{2}^{r}=0$ since all the $I_{2}^{i}$ lie in $H^{0}\left(B_{s}\left(\bar{X}, x_{0}\right)\right)$. In fact, this condition is also satisfied for $m=0$ when $s>0$.

This completes the proof that the twisted Poincare series give higher order modular forms.

For example, when $I=f$, where $f$ is a weight 2 cusp form, either holomorphic or anti-holomorphic, then $P^{2}(z, f)=-P^{3}(z, f)$ as in this case the integrals do not depend on the base point. In general, however, these forms could be different, but they are related.

In [JO06] certain higher order non-holomorphic Eisenstein series are constructed by twisting Eisenstein series by products of modular symbols. One can also twist non-holomorphic Eisenstein series by iterated integrals to get higher order non-holomorphic Eisenstein series. The ones constructed by Jorgenson and O'Sullivan are then special cases of this construction because the product of modular symbols can be expressed as a sum of iterated integrals via the shuffle product of iterated integrals.

4.1. Weight 2. The case of weight 2 modular forms requires a little more delicate handling as the Poincaré series do not converge. An approach to resolving this is to use the ideas of Diamantis and O'Sullivan [DO06]. They overcome this problem by defining it as a function obtained as a special value of the analytic continuation of a certain Poincaré series with an additional factor which makes it converge.

Precisely, for an integer $m$ and a cusp a, define

$$
\mathcal{Z}_{m, \mathbf{a}}(z, s, I)=\sum_{\gamma \in \Gamma_{\mathbf{a}} \backslash \Gamma}\left(\int_{z_{0}}^{\gamma z_{0}} I\right) \frac{e\left(m \sigma_{\mathbf{a}}^{-1} \gamma z\right) \operatorname{Im}\left(\sigma_{\mathbf{a}}^{-1} \gamma z\right)^{s}}{j\left(\sigma_{\mathbf{a}}^{-1} \gamma, z\right)^{2}} .
$$

As a function of $s$ this has an analytic continuation to the entire complex plane. In particular, one can put $s=0$ and the resulting function

$$
P_{m, \mathbf{a}}(z, I)=\mathcal{Z}_{m, \mathbf{a}}(z, 0, I)
$$

is a weight 2 , order $s+1$ modular form. The argument is similar to that of Diamantis and Sim [DS06a]. However, as the details are complicated, we will not deal with this case in the remaining part of our paper.

4.2. Spaces of higher order and higher weight modular forms. In the previous sections we constructed some examples of higher weight, higher order forms. We would like to define the space $M_{\text {geom, }}^{s+1}\left(\Gamma, x_{0}\right)$ to be the largest space we can get from the constructions above. For that we first define a space of primitive forms. 
The space of primitive cusp forms $\mathrm{SPM}_{\text {geom, } k}^{s+1}\left(\Gamma, x_{0}\right)$ is defined to be the space spanned by the forms $P_{m, \mathbf{a}}(z, I)$ and their complex conjugates over all cusps a and all positive integers $m$. The space of primitive modular forms $\mathrm{PM}_{\text {geom }, k}^{s+1}\left(\Gamma, x_{0}\right)$ is then the space spanned by $\mathrm{SPM}_{\text {geom }, k}^{s+1}$ and the Eisenstein series $E_{\mathbf{a}}(z, I)=P_{0, \mathbf{a}}(z, I)$ over all cusps a where $I$ is in $H^{0}\left(B_{s}(\bar{X}), z_{0}\right)$. As the space of smooth modular forms of order $s$ and weight $k$ is finitedimensional, this space is finite-dimensional. In weight 0 all the forms constructed above as anti-derivatives of iterated integrals are said to be primitive.

There is a product structure on the space of modular forms of higher order. This was first introduced by O'Sullivan. If $F$ is a modular form of weight $k_{1}$ and order $s_{1}$ and $G$ is a modular form of weight $k_{2}$ and order $s_{2}$, then from [CD06], using the Rankin-Cohen bracket for $N=0$, we find that $F G$ is a modular form of weight $k_{1}+k_{2}$ and order $s_{1}+s_{2}-1$.

We can see this easily in the weight 0 case: the product of two iterated integrals of orders $s_{1}$ and $s_{2}$, which correspond to modular forms of order $s_{i}+1$, is an iterated integral of order $s_{1}+s_{2}$ whose anti-derivative is a modular form of order $s_{1}+s_{2}+1=\left(s_{1}+1\right)+\left(s_{2}+1\right)-1$.

So we finally define the space of geometric higher order modular forms of order $s$ and weight $k, M_{\text {geom, } k}^{s}\left(\Gamma, x_{0}\right)$, to be the algebra generated by the primitive forms, and similarly the space of geometric higher order cusp forms $\mathrm{SM}_{\text {geom, }, k}^{s}\left(\Gamma, x_{0}\right)$ to be the subalgebra generated by the primitive cusp forms. A weight $k$ form, therefore, is a sum of products of lower weight primitive forms. To study this space in more detail we have to include the space of weight 2 higher order forms, which, as mentioned above, requires more delicate handling, so in what follows we will only consider the primitive spaces.

4.3. Hodge structures. We can define an ad hoc Hodge structure on the spaces of primitive modular forms, $\mathrm{PM}_{\text {geom }, k}^{s}$. The weight and Hodge filtrations are defined as follows. Recall that on $H^{0}\left(B_{s}(\bar{X}), x_{0}\right)$, the (Hodge) weight filtration and filtration by length coincide. Define

- $W_{l} \mathrm{PM}_{\text {geom }, k}^{s+1}=$ span of $P_{m, \mathbf{a}, k}(z, I), \bar{P}_{m, \mathbf{a}, k}(z, I)$ and $E_{\mathbf{a}, k}(z, K)$ such that $I \in W_{l-(k-1)} H^{0}\left(B_{s}(\bar{X}), x_{0}\right), m>0$ and $K \in W_{l-k} H^{0}\left(B_{s}(\bar{X}), x_{0}\right)$ and where all the Poincaré and Eisenstein series are of weight $k$.

- $F^{p} \mathrm{PM}_{\text {geom }, k^{s+1}}=$ span of $P_{m, \mathbf{a}, k}(z, I), \bar{P}_{m, \mathbf{a}, k}(z, J)$ and $E_{\mathbf{a}, k}(z, K)$ such that $m>0, I \in F^{p-(k-1)} H^{0}\left(B_{s}(\bar{X}), x_{0}\right), J \in F^{p} H^{0}\left(B_{s}(\bar{X}), x_{0}\right)$ and $K \in F^{p-k / 2} H^{0}\left(B_{s}(\bar{X}), x_{0}\right)$.

So, for example, if $k=4, s=2$, the weight filtration on $\mathrm{PM}_{\text {geom,4 }}^{3}$ is as follows: 
- $W_{0}=W_{1}=W_{2}=0$.

- $W_{3}=$ span of $P_{m, \mathbf{a}}(z)$ and $\bar{P}_{m, \mathbf{a}}(z)=$ holomorphic and anti-holomorphic cusp forms of weight 4 .

- $W_{4}=$ span of Eisenstein series $E_{\mathbf{a}}(z)$ of weight 4 and span of $P_{m, \mathbf{a}}(z, I)$ and $\bar{P}_{m, \mathbf{a}}(z, I)$, where $I$ is in $H^{0}\left(B_{1}(\bar{X}), z_{0}\right)$.

- $W_{5}=$ span of $W_{4}, P_{m, \mathbf{a}}(z, I), \bar{P}_{m, \mathbf{a}}(z, I)$ where $I$ is in $H^{0}\left(B_{2}(\bar{X}), x_{0}\right)$, and $E_{\mathbf{a}}(z, J)$ where $J$ is in $H^{0}\left(B_{1}(\bar{X}), z_{0}\right)$.

- $W_{6}=\mathrm{PM}_{\text {geom }, 4}^{3}=W_{i}, i \geq 6=\operatorname{span}$ of $W_{5}$ and $E_{\mathbf{a}}(z, J)$ where $J$ is in $H^{0}\left(B_{2}(\bar{X}), x_{0}\right)$.

The Hodge filtration is given as follows:

- $F^{0}=\mathrm{PM}_{\text {geom, } 4}^{3}$.

- $F^{1}=\operatorname{span}$ of $\bar{P}_{\mathfrak{m}, \mathbf{a}}(z, I)$ where $I \in F^{1} H^{0}\left(B_{2}(\bar{X}), x_{0}\right), P_{\mathfrak{m}, \mathbf{a}}(z, J)$ where $J \in H^{0}\left(B_{2}(\bar{X}), x_{0}\right)$, and $E_{\mathbf{a}}(z, K)$ where $K \in H^{0}\left(B_{2}(\bar{X}), x_{0}\right)$.

- $F^{2}=\operatorname{span}$ of $\bar{P}_{\mathfrak{m}, \mathbf{a}}(z, I)$ where $I \in F^{2} H^{0}\left(B_{2}(\bar{X}), x_{0}\right), P_{\mathfrak{m}, \mathbf{a}}(z, J)$ where $J \in H^{0}\left(B_{2}(\bar{X}), x_{0}\right)$, and $E_{\mathbf{a}}(z, K)$ where $K \in H^{0}\left(B_{2}(\bar{X}), x_{0}\right)$.

- $F^{3}=\operatorname{span}$ of $P_{\mathfrak{m}, \mathbf{a}}(z, J)$ where $J \in H^{0}\left(B_{2}(\bar{X}), x_{0}\right)$, and $E_{\mathbf{a}}(z, K)$ where $K \in F^{1} H^{0}\left(B_{2}(\bar{X}), x_{0}\right)$.

- $F^{4}=$ span of $P_{\mathfrak{m}, \mathbf{a}}(z, J)$ where $J \in F^{1} H^{0}\left(B_{2}(\bar{X}), x_{0}\right)$, and $E_{\mathbf{a}}(z, K)$ where $K \in F^{2} H^{0}\left(B_{2}(\bar{X}), x_{0}\right)$.

- $F^{5}=\operatorname{span}$ of $P_{\mathfrak{m}, \mathbf{a}}(z, J)$ where $J \in F^{2} H^{0}\left(B_{2}(\bar{X}), x_{0}\right)$.

- $F^{i}=0, i \geq 6$.

We expect that this Hodge structure can be used to define a Hodge structure on the full space of geometric higher order forms using the fact that it is the algebra generated by these forms, but since that requires the weight 2 case as well, we will not consider it here.

At the moment it is not clear whether there is some natural geometric structure underlying this Hodge structure, and so one cannot say anything about naturality or functoriality. However, there is some recent work of Anton Dietmar [Die08] relating higher order forms with Lie algebra cohomology, and one might hope that this Hodge structure is related to a natural Hodge structure on those cohomology groups.

Acknowledgements. I would like to thank Nikolaos Diamantis for careful reading and comments on several earlier versions of this manuscript. I would also like to thank G. V. Ravindra, J. G. Biswas, D. Pancholi and N. Fakhruddin for their comments and help. I would like to thank the Max-Planck Institute in Bonn and the TIFR Centre in Bangalore for their hospitality when this work was done. Finally, I would like to thank the referee for suggesting a considerably simpler argument for the main theorem. 


\section{References}

[Che71] K. T. Chen, Algebras of iterated path integrals and fundamental groups, Trans. Amer. Math. Soc. 156 (1971), 359-379.

[CDO02] G. Chinta, N. Diamantis, and C. O'Sullivan, Second order modular forms, Acta Arith. 103 (2002), 209-223.

[CD06] Y. Choie and N. Diamantis, Rankin-Cohen brackets on higher order modular forms, in: Multiple Dirichlet Series, Automorphic Forms, and Analytic Number Theory, Proc. Sympos. Pure Math. 75, Amer. Math. Soc., Providence, RI, 2006, 193-201.

[Del71] P. Deligne, Théorie de Hodge. II, Inst. Hautes Études Sci. Publ. Math. 40 (1971), 5-57.

[DG05] P. Deligne and A. B. Goncharov, Groupes fondamentaux motiviques de Tate mixte, Ann. Sci. École Norm. Sup. (4) 38 (2005), 1-56.

[DKMO06] N. Diamantis, M. Knopp, G. Mason, and C. O'Sullivan, L-functions of second-order cusp forms, Ramanujan J. 12 (2006), 327-347.

[DO06] N. Diamantis and C. O'Sullivan, The dimension of spaces of holomorphic second-order cusp forms and their cohomology, preprint, 2006.

[DS06a] N. Diamantis and D. Sim, The classification of spaces of higher order cusp forms, preprint, 2006.

[DS06b] N. Diamantis and R. Sreekantan, Iterated integrals and higher order automorphic forms, Comment. Math. Helv. 81 (2006), 481-494.

[Die08] A. Dietmar, Higher order cohomology of arithmetic groups, arXiv:0805. 0703v2 [math.NT], 2008.

[Gol99] D. Goldfeld, Zeta functions formed with modular symbols, in: Automorphic Forms, Automorphic Representations, and Arithmetic (Fort Worth, TX, 1996), Proc. Sympos. Pure Math. 66, Amer. Math. Soc., Providence, RI, 1999, Part 1, 111-121.

[GO03] D. Goldfeld and C. O'Sullivan, Estimating additive character sums for Fuchsian groups, Ramanujan J. 7 (2003), 241-267.

[Hai87] R. M. Hain, The geometry of the mixed Hodge structure on the fundamental group, in: Algebraic Geometry, Bowdoin, 1985 (Brunswick, ME, 1985), Proc. Sympos. Pure Math. 46, Amer. Math. Soc., Providence, RI, 1987, Part 2, $247-282$.

[JO06] J. Jorgenson and C. O'Sullivan, Unipotent vector bundles and higher-order non-holomorphic Eisenstein series, preprint, 2006.

[KZ03] P. Kleban and D. Zagier, Crossing probabilities and modular forms, J. Statist. Phys. 113 (2003), 431-454.

[Sar90] P. Sarnak, Some Applications of Modular Forms, Cambridge Tracts in Math. 99, Cambridge Univ. Press, Cambridge, 1990.

Indian Statistical Institute, Bangalore Centre

8th Mile, Mysore Road, RV College Post

Jnana Bharathi, Bangalore

Karnataka 560059, India

E-mail: rameshsreekantan@gmail.com 